\title{
Lung clearance index to monitor treatment response in pulmonary exacerbations in preschool children with cystic fibrosis
}

\author{
Jonathan H Rayment, ${ }^{1,2}$ Sanja Stanojevic, ${ }^{1,2}$ Stephanie D Davis, ${ }^{3}$ \\ George Retsch-Bogart, ${ }^{4}$ Felix Ratjen ${ }^{1,2,5}$
}

- Additional material is published online only. To view please visit the journal online (http://dx.doi.org/10.1136/ thoraxjnl-2017-210979).

${ }^{1}$ Division of Respiratory Medicine, Hospital for Sick Children, Toronto, Ontario, Canada

${ }^{2}$ Department of Paediatrics, University of Toronto, Toronto, Ontario, Canada

${ }^{3}$ Section of Pediatric

Pulmonology, Allergy and Sleep Medicine, Department of Pediatrics, Riley Hospital for Children, Indiana University School of Medicine, Indianapolis, Indiana, USA

${ }^{4}$ Division of Pediatric

Pulmonology, University of North Carolina at Chapel Hill,

Chapel Hill, North Carolina, USA

${ }^{5}$ Translational Medicine

Program, SickKids Research

Institute, Toronto, ON, Canada

\section{Correspondence to} Dr Jonathan H Rayment, Division of Respiratory Medicine, British Columbia Children's Hospital, 4480 Oak St, Vancouver, BC V6H 3N1, Canada;

jonathan.rayment@cw.bc.ca

Received 31 August 2017 Revised 4 December 2017

Accepted 22 January 2018

Published Online First

15 February 2018

\begin{abstract}
Background Antibiotic treatment for pulmonary symptoms in preschool children with cystic fibrosis (CF) varies among clinicians. The lung clearance index ( $\mathrm{LCl}$ ) is sensitive to early CF lung disease, but its utility to monitor pulmonary exacerbations in young children has not been assessed.

Objective We aim to (1) understand how $\mathrm{LCl}$ changes during lower respiratory tract symptoms relative to a recent clinically stable measurement, (2) determine whether $\mathrm{LCl}$ can identify antibiotic treatment response and (3) compare $\mathrm{LCl}$ changes to changes in spirometric indices.
\end{abstract}

Methods $\mathrm{LCl}$ and spirometry were measured at quarterly clinic visits over a 12-month period in preschool children with CF. Symptomatic visits were identified and classified as treated or untreated. Treatment response was estimated using propensity score matching methods. Results 104 symptomatic visits were identified in 78 participants. $\mathrm{LCl}$ increased from baseline in both treated (mean relative change $+23.8 \%(95 \% \mathrm{Cl} 16.2$ to 31.4$)$ ) and untreated symptomatic visits (mean relative change $+11.2 \%(95 \% \mathrm{Cl} 2.4$ to 19.9$)$ ). A significant antibiotic treatment effect was observed when $\mathrm{LCl}$ was used as the outcome measure (average treatment effect $-15.5 \%$ (95\% Cl -25.4 to -5.6$)$ ) but not for z-score FEV.

Conclusion $\mathrm{LCl}$ significantly deteriorated with pulmonary symptoms relative to baseline and improved with antibiotic treatment. These data suggest that $\mathrm{LCl}$ may have a role in the routine clinical care of preschool children with CF.

\section{INTRODUCTION}

Lung disease remains the major cause of morbidity and mortality in patients with cystic fibrosis (CF). Acute pulmonary exacerbations (PEx) treated with intravenous antibiotics are associated with a faster rate of pulmonary function decline and worse 5 -year survival. ${ }^{2-4}$ Mild exacerbations, treated with outpatient oral antibiotics, are also associated with worse long-term lung function outcomes. ${ }^{5-7} \mathrm{PEx}$ events often present as non-specific worsening of respiratory symptoms, such as increased cough or sputum production, and the decision to treat with antibiotics is commonly supported by a precipitous drop in lung function, typically measured by spirometry and assessed through the forced expiratory volume in one second $\left(\mathrm{FEV}_{1}\right){ }^{8}{ }^{9}$ Treatment decisions are particularly challenging in young
Key messages

What is the key question?

- Can the lung clearance index (LCI) help clinicians diagnose pulmonary exacerbations in preschool children with cystic fibrosis and monitor response to treatment?

What is the bottom line?

- $\mathrm{LCl}$ is more sensitive than spirometry in detecting lower respiratory tract symptoms in this population and can also detect a response to antibiotic treatment where spirometry cannot.

Why read on?

- These data are important for the potential integration of $\mathrm{LCl}$ into clinical testing for preschool children with cystic fibrosis.

children with $\mathrm{CF},{ }^{10}$ as increased cough, which is the most common symptom of a PEx in mild CF lung disease, ${ }^{11}$ is often the result of viral upper respiratory tract infections, ${ }^{12}$ and spirometry is not routinely performed in this population. The diagnosis and treatment of PEx in young children vary significantly among clinicians ${ }^{13-15}$; therefore, new objective measures of lung function are needed to aid in the management of PEx events in this population.

The lung clearance index (LCI) is a measure of ventilation inhomogeneity acquired from multiple breath washout (MBW) testing ${ }^{16}$ and is more sensitive than $\mathrm{FEV}_{1}$ at detecting lung disease in $\mathrm{CF}^{17}{ }^{18}$ In particular, LCI measured during the preschool years is predictive of both LCI and FEV measured $_{1}$ at school age. ${ }^{19}$ The LCI also improves after treatment with intravenous antibiotics for PEx events, although the response is heterogeneous. ${ }^{20-25}$ This varied treatment response may be related to the degree of worsening of LCI with symptoms, as has been shown with $\mathrm{FEV}_{1}{ }^{26}{ }^{27}$ However, previous studies did not incorporate baseline LCI measurements taken at clinically relevant intervals and did not address this question.

Recently, we reported that lower respiratory tract (LRT) symptoms in preschool children with CF, but not in healthy children, and PEx in CF (defined as increased LRT symptoms treated with antibiotics) 
were associated with higher LCI values. ${ }^{28}$ Although these findings provided important information regarding the relationship between clinical symptoms and objective measures of ventilation inhomogeneity, the clinical utility of this test to monitor lung function during PEx and to track response to treatment have yet to be addressed.

In this study, we aim to (1) understand how LCI changes during LRT symptoms relative to a recent clinically stable measurement, (2) determine whether LCI can identify antibiotic treatment response and (3) compare LCI changes to changes in spirometric indices.

\section{METHODS}

\section{Study design}

This is a prospective multicentre observational study in preschool children with $\mathrm{CF}$ and age-matched healthy controls between the ages of 2.5 years and 6 years. ${ }^{28}$ Children had LCI measured at time points that represent routine clinical follow-up (enrolment, 1 month, 3 months, 6 months, 9 months and 12 months). In the current study, participants with CF were asked to attend extra study visits during episodes of acutely worsened LRT symptoms (increased cough, wheeze, sputum production or shortness of breath) regardless of whether they received antibiotic treatment for these symptoms.

\section{Exposure definitions}

Documentation for all visits, including both study and clinical notes, was reviewed independently by two of the authors (JHR and FR) to classify the visits as symptomatic or asymptomatic based on participant/parent reported symptoms in addition to physical examination. Disagreements between reviewers were discussed, and consensus was achieved in all cases. Symptomatic visits were defined as those where LRT symptoms (cough, wheeze, sputum production or shortness of breath) or signs on physical exam (work of breathing, hypoxaemia, tachypnoea, wheeze, crackles and differential air entry) were judged to be increased from the participant's baseline. Symptomatic visits were further categorised as PEx events if new oral, inhaled or intravenous antibiotic therapy was initiated by the treating physician at the time of the symptomatic visit (treatment medication and modality were decided by the treating physician).

On some occasions, antibiotic therapy was initiated by the treating physician before the study visit (eg, prescription called in from the clinic or provided by a primary care practitioner). In these cases, the visit was classified as being preceded by an unobserved PEx (uPEx). If the participant attended a study visit while continuing antibiotics that had been prescribed for a uPEx, this visit was categorised both as a treated, symptomatic visit (PEx) and as being preceded by a uPEx. Visits where the participant was on antibiotics prescribed for a uPEx event but had no increased LRT symptoms were excluded from the analysis.

Baseline visits were defined as the most recent asymptomatic visit prior to the study visit. Follow-up visits were defined as the next chronological study visit, regardless of symptom classification. A schematic of visit definitions is shown in online supplementary figure S1.

\section{Outcomes}

Multiple breath nitrogen washout testing was performed on the Exhalyzer D (EcoMedics, Dürnten, Switzerland) with modifications for preschool children as previously described. ${ }^{28}$ LCI was reported if at least two technically acceptable trials were collected at a visit. Acceptability criteria were implemented as previously described. ${ }^{29}$ Preschool spirometry was performed according to American Thoracic Society/European Respiratory Society guidelines. ${ }^{30}$ Percent-predicted values and z-scores for $\mathrm{FEV}_{1}$ (or forced expiratory volume in $0.75 \mathrm{~s}\left(\mathrm{FEV}_{0.75}\right)$ if the child was unable to achieve a forced exhalation for $1 \mathrm{~s}$ ) were calculated using Global Lung Function Initiative reference equations and reported as ppFEV and zFEV, respectively. ${ }^{31}$

Although treating clinicians were not blinded to the spirometry or MBW results, study results were not routinely shared. Spirometry is occasionally performed in the preschool age group at all sites but not part of routine practice.

Changes between visits in PFT or LCI outcomes are shown as either absolute change, measured in units (eg, a 1.3 LCI unit drop between visits) or as relative change, as a per cent of the preceding measurement (eg, a drop from a ppFEV of $80 \%$ to $70.4 \%$ between visits represents a $-12 \%$ relative change in ppFEV).

\section{Statistical analysis}

All statistical analyses were performed in Stata V.14 (StataCorp, College Station, TX, USA) . Group differences between participants with and without a PEx event during the study were compared using t-tests for normally distributed continuous variables and Wilcoxon rank-sum test for non-normally distributed continuous variables. The change in LCI and zFEV between baseline and symptoms and between symptoms and follow-up were compared with linear regression using generalised estimating equations (GEE) model to account for repeated measurements in the same participant. Sensitivity analyses included only the first symptomatic event for each participant and excluded visits preceded by uPEx events. Participant characteristics associated with the change in LCI between baseline and symptoms and between symptoms and follow-up were also investigated with a linear regression-GEE model.

Based on recently published reproducibility data for LCI and preschool spirometry in this dataset, changes in LCI and zFEV between visits were separated into those that showed a significant change between visits, using relative increase or decrease in LCI of $15 \%$ and a decrease of $1.3 \mathrm{z}$-scores for $\mathrm{zFEV}^{32}$ Further details are available in the online supplement.

To estimate the treatment effect of antibiotic therapy, the percentage change in LCI between the symptomatic and follow-up visits were compared between treated and untreated visits using propensity score matching analysis (-teffects- and -psmatch- functions in Stata). This approach allows for the estimation of the average treatment effect on the treated group, where the treated and untreated groups are matched for baseline characteristics. Each treated visit was matched with an untreated visit using probabilistic matching based on covariates related to the outcome regardless of their exposure; we included LCI at baseline, the relative change in LCI from baseline and age at symptoms. Sensitivity analysis limited to those visits with a significant worsening of LCI greater than $+15 \%$.

\section{RESULTS}

Three hundred and seventy-two visits in 78 participants with CF were included in this analysis, of which 104 visits were symptomatic (figure 1, online supplementary figure S2). Of the 78 participants, $62(79.5 \%)$ had at least one episode of increased LRT symptoms over the 12-month period (median events per participant 1 (range $0-7)$ ). The 62 participants who experienced LRT symptoms during the study were similar in demographic characteristics, medical history, medication use 


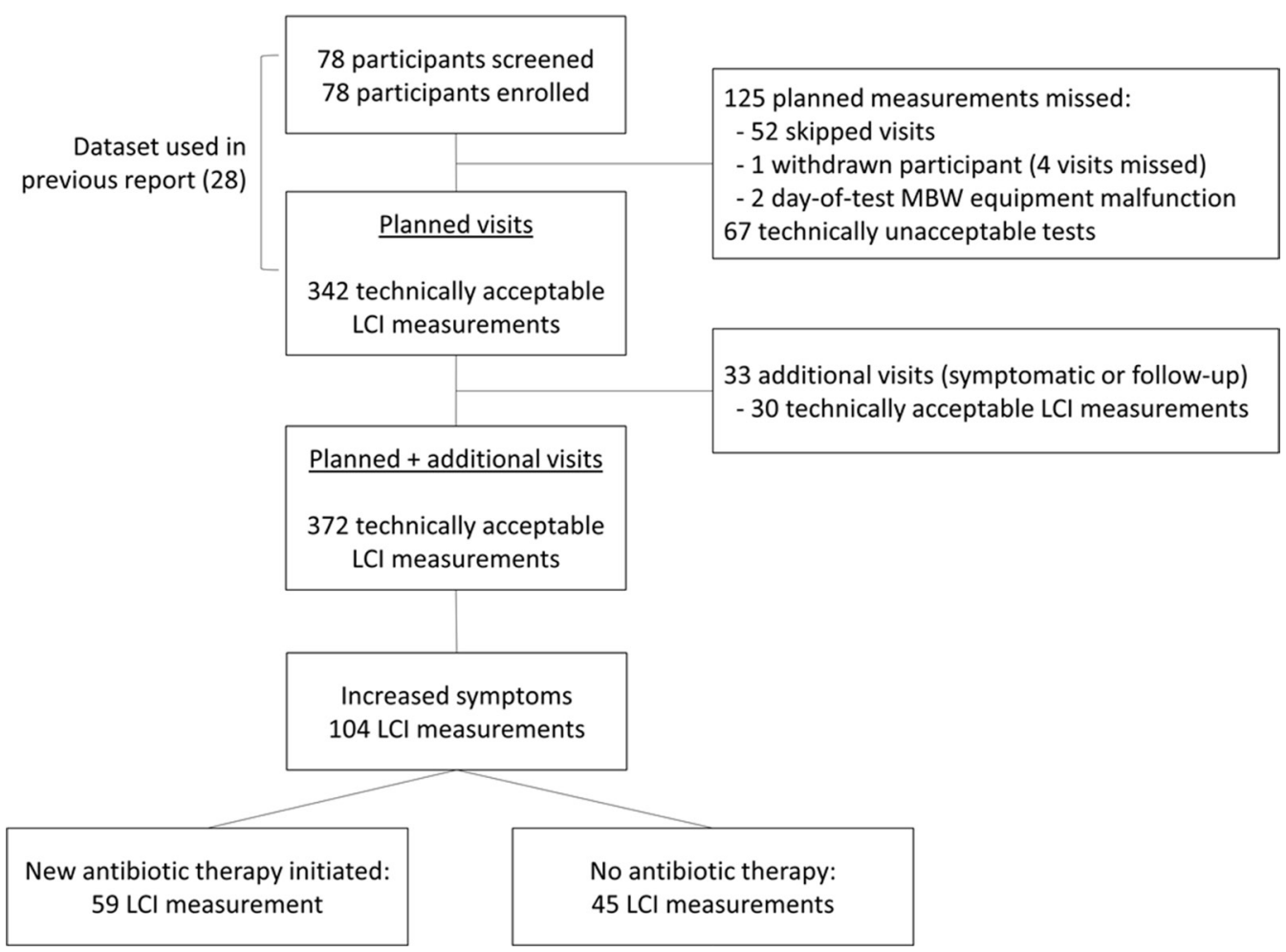

Figure 1 Flow diagram outlining participant enrolment, visit summary and study groups. LCl, lung clearance index; MBW, multiple breath washout.

Table 1 Comparison of participants who had at least one symptomatic event during the 12-month study period to those who had none

\begin{tabular}{|c|c|c|c|c|c|}
\hline & $\mathrm{n}$ & $\begin{array}{l}\text { Participants with at least one } \\
\text { symptomatic episode }(n=62)\end{array}$ & n & $\begin{array}{l}\text { Participants with no documented } \\
\text { symptomatic episode }(n=16)\end{array}$ & p \\
\hline Age at enrolment & 62 & $4.4(3.5-5.2)$ & 16 & $3.8(3.6-5.2)$ & 0.63 \\
\hline Male sex & 62 & $32(51.6)$ & 16 & $7(43.8)$ & 0.39 \\
\hline $\mathrm{LCl}$ at enrolment & 43 & $8.8(8.3-9.3)$ & 8 & $8.6(7.0-10.2)$ & 0.71 \\
\hline $\mathrm{zFEV}_{1}$ at enrolment & 27 & $-0.41(-1.1$ to 0.1$)$ & 3 & $-0.41(-1.4$ to 0.43$)$ & 0.54 \\
\hline BMI centile* at enrolment & 62 & $48.0(40.1-55.0)$ & 16 & $45.4(33.2-57.6)$ & 0.73 \\
\hline \multicolumn{6}{|l|}{ Genotype } \\
\hline Class I-III & 62 & $60(96.8)$ & 16 & $15(93.8)$ & 0.50 \\
\hline Class IV-V & 62 & $2(3.2)$ & 16 & $1(6.3)$ & 0.50 \\
\hline \multicolumn{6}{|l|}{ Maintenance therapy at enrolment } \\
\hline \multicolumn{6}{|c|}{ Sputum/oropharyngeal swab microbiology (ever positive in 12 months prior to study) } \\
\hline Methicillin sensitive Staphylococcus aureus & 62 & $55(88.7)$ & 16 & $13(81.3)$ & 0.42 \\
\hline Pseudomonas aeruginosa & 62 & $38(61.3)$ & 16 & $8(50.0)$ & 0.57 \\
\hline Haemophilus influenzae & 62 & $33(53.2)$ & 16 & $7(43.8)$ & 0.58 \\
\hline Stenotrophomonas maltophilia & 62 & $13(21.0)$ & 16 & $3(18.8)$ & 1.00 \\
\hline Methicillin-resistant Staphylococcus aureus & 62 & $10(16.1)$ & 16 & $1(6.3)$ & 0.44 \\
\hline Burkholderia cepacia complex & 62 & $0(0)$ & 16 & $2(12.5)$ & 0.04 \\
\hline
\end{tabular}

Data are presented as median (IQR) or number (\%) as appropriate unless otherwise stated. P values for Wilcoxon rank-sum or Fisher's exact tests are shown.

*Based on CDC growth chart.

Data acquired at the enrolment visit are shown.

$\mathrm{BMI}$, body mass index; $\mathrm{LCl}$, lung clearance index; $\mathrm{zFEV}_{1}, \mathrm{z}$-score for $\mathrm{FEV}_{1}$. 
Table 2 Characteristics of the treated and untreated symptomatic visits

\begin{tabular}{|c|c|c|c|}
\hline & Treated $(n=59)$ & Untreated $(n=45)$ & $\mathrm{p}$ \\
\hline Age at time of symptoms (years)* & $4.7(4.3-5.6)$ & $4.8(4.1-5.5)$ & 0.99 \\
\hline \multicolumn{4}{|l|}{ Route of treatment $(\mathrm{n}(\%))$} \\
\hline Oral & $48(81.4)$ & - & \\
\hline Inhaled & $3(5.1)$ & - & \\
\hline Intravenous inpatient & $8(13.6)$ & - & \\
\hline \multicolumn{4}{|l|}{ Missing visits† (n (\%)) } \\
\hline Missing baseline only & $15(25.4)$ & $13(28.9)$ & 0.24 \\
\hline Missing follow-up only & $9(15.3)$ & $12(26.7)$ & \\
\hline Missing neither baseline nor follow-up & $35(59.3)$ & $20(44.4)$ & \\
\hline \multicolumn{4}{|l|}{ Time intervals (days)* } \\
\hline Baseline to symptomatic visit & $109(84-162)$ & $112(84-184)$ & 0.94 \\
\hline Symptomatic visit to follow-up & $72(35-98)$ & $91(43-98)$ & 0.33 \\
\hline \multicolumn{4}{|l|}{ Pulmonary function at symptomatic visitł; mean $(95 \% \mathrm{Cl})$} \\
\hline LCI (units) (treated $n=59$, untreated $n=45$ ) & $10.4(9.8$ to 11.1$)$ & $9.8(9.1$ to 10.4$)$ & 0.001 \\
\hline zFEV (z-scores) (treated $n=44$, untreated $n=33$ ) & $-1.4(-1.8$ to -1.0$)$ & $-0.6(-1.0$ to -0.2$)$ & $<0.001$ \\
\hline ppFEV (\%) (treated $n=44$, untreated $n=33$ ) & $79.8(74.3$ to 85.4$)$ & $91.8(86.0$ to 97.5$)$ & $<0.001$ \\
\hline \multicolumn{4}{|c|}{ Change in pulmonary function from baseline to symptomatic visitł; mean difference $(95 \% \mathrm{Cl})$} \\
\hline Relative $\mathrm{LCl}$ change $(\%)$ (treated $\mathrm{n}=44$, untreated $\mathrm{n}=32$ ) & $23.8(16.2$ to 31.4$)$ & $11.2(2.4$ to 19.9$)$ & 0.02 \\
\hline Absolute $L C I$ change (units) (treated $n=44$, untreated $n=32$ ) & $1.8(1.2$ to 2.3$)$ & $0.9(0.2$ to 1.5$)$ & 0.03 \\
\hline Relative ppFEV change (\%) (treated $n=34$, untreated $n=19$ ) & $-9.3(-14.7$ to -3.9$)$ & $3.4(-3.5$ to 10.3$)$ & 0.002 \\
\hline Absolute zFEV change ( $z$-scores) (treated $n=34$, untreated $n=19$ ) & $-0.7(-1.0$ to -0.3$)$ & $0.2(-0.3$ to 0.6$)$ & 0.003 \\
\hline \multicolumn{4}{|l|}{ Average treatment effect; mean difference $(95 \% \mathrm{Cl}) \S$} \\
\hline Relative change in $\mathrm{LCl}(\%)$ & \multicolumn{2}{|c|}{$-15.5(-25.4$ to -5.6$)$} & 0.002 \\
\hline Absolute change in zFEV (z-scores) & \multicolumn{2}{|c|}{$0.58(-0.30$ to 1.47$)$} & 0.20 \\
\hline Relative change in ppFEV (\%) & \multicolumn{2}{|c|}{$1.8(-17.2$ to 20.8$)$} & 0.19 \\
\hline
\end{tabular}

*Data presented as median (IQR). P value shown for Wilcoxon rank-sum test for comparison of medians.

tP value for $\chi^{2}$ test of association is shown.

‡Data presented as estimated mean values $(95 \% \mathrm{Cl})$ as determined by linear regression using $\mathrm{GEE}$ models to account for multiple measurements in the same participant.

IMixed effects linear regression model results shown as convergence not achieved for GEE model.

$\S$ Average treatment effect estimated using propensity score matching, accounting for baseline parameter value and magnitude of worsening parameter from baseline at the time of symptoms. Results shown as mean relative change in $\mathrm{LCl}$ and mean absolute change in zFEV.

$\mathrm{GEE}$, generalised estimating equations; $\mathrm{LCl}$, lung clearance index; $\mathrm{ppFEV}$, per cent-predicted values for $\mathrm{FEV}_{1} ; \mathrm{zFEV}_{1}, \mathrm{z}-\mathrm{score}$ for $\mathrm{FEV}_{1}$.

and microbiology, to the 16 who did not experience LRT symptoms (table 1).

There were 74 uPEx events reported. Of those, 48 had completed the prescribed treatment course by the study visit and 26 were still taking antibiotics. Of those 26 still taking antibiotics, four reported that their symptoms had completely resolved and were therefore excluded from analysis.

In the 104 symptomatic visits, antibiotic therapy was either continued or initiated by the treating physician in 59 visits $(56.7 \%)$, while no treatment was given in 45 visits (table 2). Antibiotic therapy was most commonly administered as outpatient oral treatment $(81.4 \%)$. The time between the baseline visit and symptomatic visit was similar between the treated and untreated group, as was the proportion of visits with incomplete data (table 2). At the symptomatic visits, participants who received antibiotic treatment had a higher LCI (mean difference 0.7 units (95\% CI 0.3 to 1.1)) and lower zFEV (mean difference $-0.8(95 \%$ CI -1.1 to -0.6$)$ ) than those who did not receive therapy (table 2).

The group changes in LCI from baseline to symptoms and from symptoms to follow-up for treated and untreated visits are presented in figure $2 \mathrm{~B}$. LCI increased (deteriorated) with symptoms from baseline values in both the treated and untreated groups; the LCI deterioration was greater for those in whom antibiotic treatment was initiated (mean difference in relative change $12.6 \%$ (95\%CI 1.9 to 23.3 ), $\mathrm{P}=0.02$; table 2 ). At the follow-up visit, the treated group had an improvement in LCI relative to the symptomatic visit, whereas the untreated group did not.

Participants with lower LCI values at baseline had a greater deterioration between baseline and symptoms (slope -5.9 (95\% CI -8.8 to -3.0$)$ ). Furthermore, a greater relative LCI improvement at follow-up was seen in those participants with a greater worsening of LCI with symptoms (slope -0.5 (95\% CI -0.6 to -0.4$)$ ). Finally, participants who received antibiotic treatment had a greater relative improvement than those who did not receive antibiotics $(-14.1$ (95\% CI -23.0 to -5.3$))$. No other participant characteristics were associated with either LCI deterioration with symptoms or LCI improvement at follow-up.

After matching treated participants with untreated participants based on baseline characteristics, there was a significant improvement in LCI observed in the treated group relative to the untreated group (average treatment effect $-15.5 \%(95 \% \mathrm{CI}$ -25.4 to -5.6$)$ ). These results demonstrate that LCI improves 
A

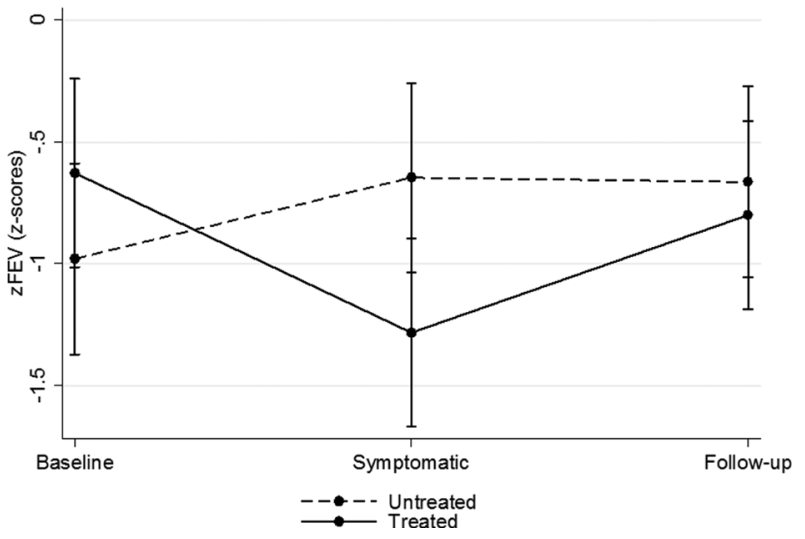

B

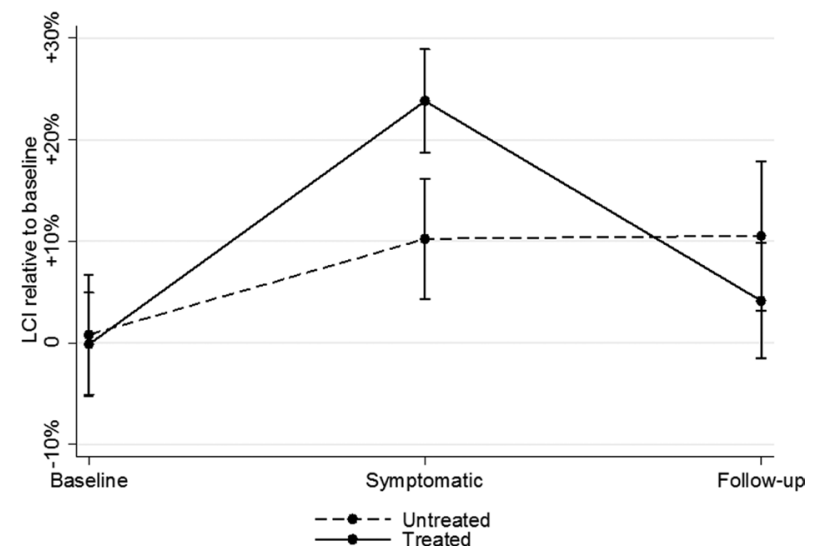

Figure 2 Change in LCl and zFEV between study timepoints. Mean absolute values of zFEV (A) and mean relative values of $\mathrm{LCl}$ (B) at baseline, symptomatic and follow-up visits for treated (solid line) and untreated (dashed line) symptomatic visits. Data points and the $95 \% \mathrm{Cl}$ (vertical bars) are estimated from linear regressions using a generalised estimating equation model to account for multiple measurements in the same participant. $\mathrm{LCl}$, lung clearance index; zFEV, z-score for $\mathrm{FEV}_{1}$.

with antibiotic treatment, independent of the differences in baseline characteristics between the groups (table 2). The treatment effect was not observed using zFEV or ppFEV. In a subset of visits with a significant worsening of LCI, the magnitude of the LCI treatment effect was similar (average treatment effect -14.2 (95\% CI -25.2 to -3.3$)$ ). Results were consistent with the main analysis when any visits in which antibiotic therapy was initiated prior to the study visit (uPEx events; $n=26$ ) were excluded or if the analysis was limited to the first symptomatic event for each participant $(n=53)$ or to those who only received oral therapy $(\mathrm{n}=48)$ (supplementary table S1).

Of the 55 symptomatic visits with both baseline and follow-up measurements, LCI deteriorated by more than $15 \%$ in approximately half $(28 / 55 ; 50.9 \%)$; of these, 23 were treated and 5 were untreated. We performed sensitivity analyses limiting to those visits where LCI worsened significantly with symptoms and where both baseline follow-up values were available. In this group, LCI improved at follow-up in $78.3 \%(18 / 23)$ of the treated participants, compared with none $(0 / 5,0 \%)$ of those who were untreated (figure 3).

zFEV decreased during symptoms and improved at follow-up in the treated group (table 2), but there was no change in zFEV across the three visits in the untreated group (figure $2 \mathrm{~A}$,). The changes in LCI and zFEV from baseline to symptoms between treated and untreated visits were assessed for the 53 visits in which measurements were available for both LCI and zFEV

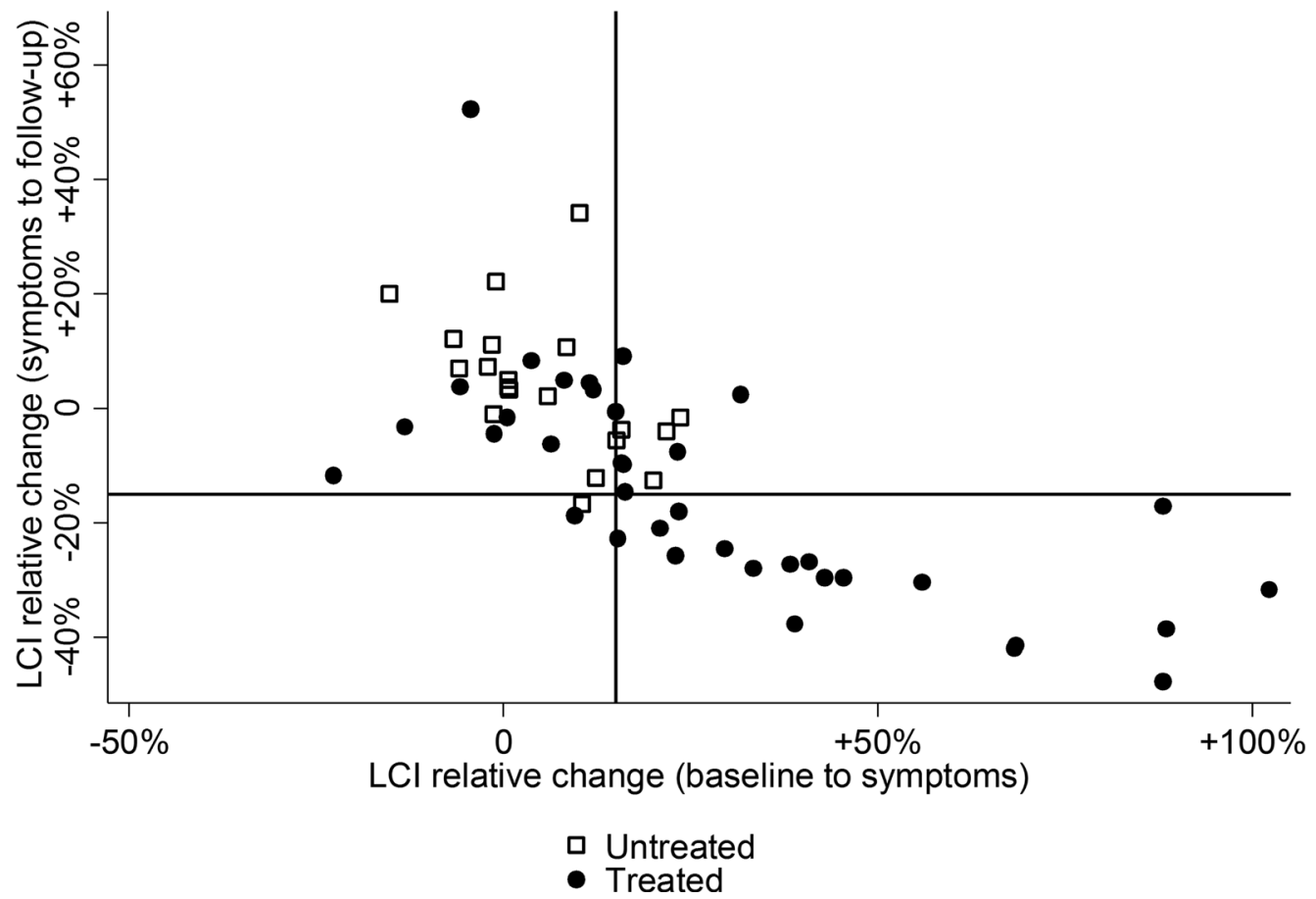

Figure 3 Comparison of $\mathrm{LCl}$ changes. Relative change in $\mathrm{LCl}$ from baseline to symptoms plotted against relative change in $\mathrm{LCl}$ from symptoms to follow-up for treated (filled circle) and untreated (hollow square) events. Lines represent thresholds for $15 \%$ relative change in $\mathrm{LCI}$. The right upper quadrant represents visits where $\mathrm{LCl}$ worsened with symptoms but failed to improve at the follow-up visit. The bottom right quadrant represents visits where $\mathrm{LCl}$ worsened with symptoms and improved at follow-up. $\mathrm{LCl}$, lung clearance index. 


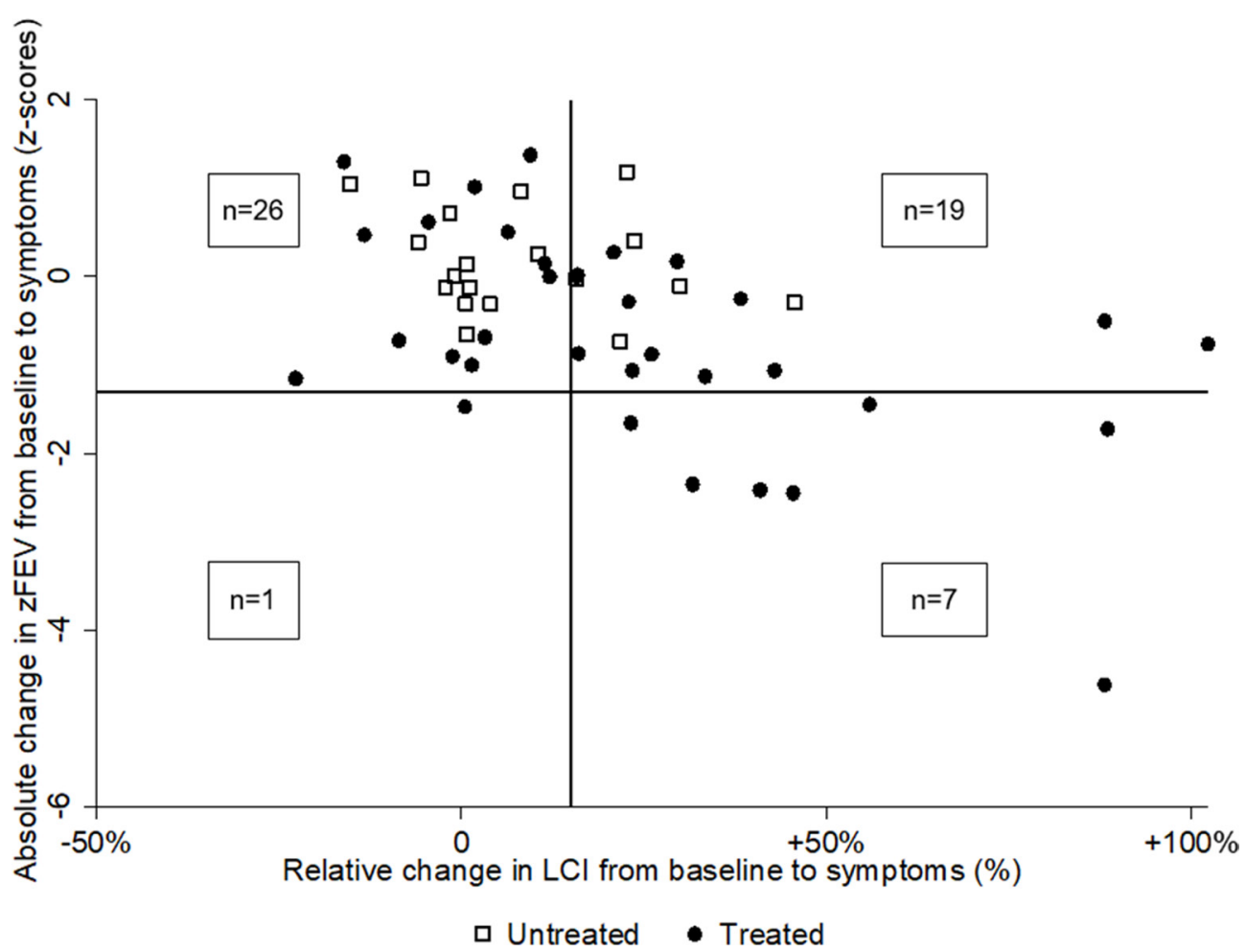

Figure 4 Comparison of changes in $\mathrm{LCl}$ and zFEV. Absolute change in zFEV between baseline and symptoms against relative change in LCl between baseline and symptoms for treated (filled circle) and untreated (hollow square) visits. Vertical line represents $15 \%$ worsening in LCl and horizontal line represents $-1.3 \mathrm{zFEV}$. The top left quadrant includes visits where neither zFEV nor LCI worsened. The bottom right quadrant includes visits where both $\mathrm{zFEV}$ and $\mathrm{LCl}$ worsened. The top right quadrant represents visits where $\mathrm{LCl}$ worsened but zFEV did not. The bottom left quadrant represents visits where zFEV worsened but $\mathrm{LCl}$ did not. $\mathrm{LCl}$, lung clearance index; zFEV, z-score for $\mathrm{FEV}_{1}$.

(figure 4). LCI worsened (>15\% percentage change from baseline) in approximately half $(26 / 53,49.1 \%)$ of the visits and $\mathrm{zFEV}$ worsened $(>1.3 \mathrm{z}$-score change from baseline) in only $7.4 \%(8 / 53)$ of the visits. Both LCI and zFEV significantly worsened in $7 / 53(13.2 \%)$ and neither significantly worsened in $26 / 53(49.1 \%)$ symptomatic visits. LCI worsened alone in $19 / 53(35.8 \%)$ visits and zFEV worsened alone in only one visit (1.9\%). In symptomatic visits where zFEV decreased signicantly, treatment was always given $(8 / 8,100 \%)$, whereas antibiotic treatment was initiated in only three quarters $(20 / 26 ; 76.9 \%)$ of visits where LCI increased significantly.

\section{DISCUSSION}

In this study, we show that the LCI increases relative to a recent clinically stable visit in preschool CF participants with increased LRT symptoms, and the LCI is more sensitive than spirometry in detecting worsening of lung function at the time of increased LRT symptoms. This was seen in participants with untreated symptoms as well as those treated with antibiotics; however, the increase was more pronounced in treated participants. Improvement in the LCI at follow-up was only observed in the treated group, and there was a treatment effect of antibiotic therapy, which was independent of baseline characteristics. Taken together, these data support a potential role for LCI in routine monitoring and clinical decision making for the preschool CF population.

On a group level, the increase in LCI from baseline with the presence of LRT symptoms was greatest in treated symptomatic participants, suggesting that the clinical judgement of 'severity' (ie, need for treatment) was reflected by a greater worsening in this objective measure of lung function. However, worsening of
LCI on a group level was also observed in symptomatic preschool children in whom the clinical decision was made not to treat, suggesting that LCI could provide additional information to the clinician to aid in treatment decisions. Consistent with the literature demonstrating that LCI is more sensitive than spirometry in detecting pulmonary pathology in $\mathrm{CF},{ }^{17-19}$ we found that LCI worsened more frequently with LRT symptoms compared with zFEV. LCI identified 19 events with a deterioration from baseline where treatment was initiated, and an additional six where treatment was not initiated; spirometry only identified eight of these events. This suggests that, on a visit-to-visit basis, LCI can provide additional information on interval lung function decline that is not detected by spirometry. In clinical practice, the use of pulmonary function testing results in isolation is unlikely, but the current study supports the concept that LCI could serve as an adjunct to clinical and spirometric assessment in preschool children with CF.

We demonstrate a treatment benefit, as measured by LCI, of antibiotics for LRT symptoms in preschool children with CF. Since this was an observational study, direct comparison of the treated participants with the untreated group was complicated, as the magnitude of LCI worsening was greater in the treated group. The improvements in the treated group may reflect regression to a mean and not necessarily the effect of the antibiotic treatment per se. To address this, we used propensity score matching to estimate the treatment effect of antibiotic treatment while accounting for the differences between the treated and untreated groups in baseline LCI and the magnitude of LCI worsening at the time of symptoms. Propensity score matching is more robust with large sample sizes; therefore, caution in interpreting these results is warranted. Ultimately, a controlled 
interventional trial is the best strategy to evaluate whether LCI can be used to monitor treatment response to antibiotic therapy.

A number of studies have previously investigated the effect of treatment of PEx on the LCI. ${ }^{20-25}$ However, the design of the current study differs on a number of fronts. First, we prospectively collected baseline measurements during periods of stability at clinically relevant intervals prior to most PEx events. One previous study did include baseline measurements in its analysis of LCI change with PEx events, ${ }^{25}$ but the baselines used were annually collected and did not necessarily reflect a recent value. Since we have shown that LCI increases in patients with CF over a 12 -month period ${ }^{28}$ annual testing may not be sufficient to capture an appropriate baseline for clinical application. In addition, the current study also included symptomatic visits where treatment was not administered. Including this untreated group allowed us to demonstrate the treatment effect of antibiotic therapy and provided important information on the natural history of LCI during and after untreated LRT symptoms. Finally, this study included mostly mild PEx events, treated with outpatient oral antibiotics, which represent the majority of exacerbations in this age group. ${ }^{14}{ }^{15}$ Taken together, these results are more applicable for the use of LCI in routine management of mild PEx events than those that were presented in previous studies.

Relative improvement in LCI at follow-up was much greater in this study than previous reports; we report $-9.7 \%$ relative change at follow-up compared with $-2.5 \%$ reported by Sonneveld $e t a l$, in a pooled analyses of studies. ${ }^{21}$ This was somewhat surprising since, unlike the previous studies, the majority of our participants had milder exacerbations, treated with outpatient oral antibiotics. The population in the current study differs from previous investigations, with a younger median age and better baseline lung function that may contribute to this finding. We also observed a negative relationship between baseline LCI and the relative increase in LCI, suggesting that participants with milder baseline disease (lower LCI) show a more pronounced increase in LCI when they are symptomatic and greater improvement with treatment. This observation is consistent with the theory that patients with more advanced lung disease show more heterogeneous response in LCI after treatment for a PEx due to shifting mucus plugging and the opening of poorly ventilated lung units. ${ }^{33} 34$ These data suggest that younger patients with milder disease may benefit more from LCI as a clinical tool in this context than older patients with more severe lung disease in whom the LCI signal is more variable.

Although this study represents a large prospective population, there are several methodological limitations. The overall sample size with complete data is limited, and missing data may not be completely missing at random; both factors may have biased the results from the statistical methods used. While every attempt was made to capture all symptomatic events, those occurring between visits may have been missed, and the number of symptom-free participants may have been overestimated. The definition of terms (eg, LRT symptoms, PEx and baseline) and the lack of prospective collection of symptom duration could also have impacted these results. The lack of standardised PEx definitions ${ }^{89}$ leads to subjectivity in physician decision making. Time to follow-up after the identification of LRT symptoms was not standardised and could have impacted the observed changes at the follow-up timepoint. Preschool spirometry is performed at some of the participating sites, which may have also influenced the decision to initiate treatment. Outcomes were based on unstandardised clinical treatment decisions (antibiotic choice, route and duration), and thus the results may have been confounded by indication. The treatment effect observed in LCI was attributed to antibiotic therapy, but treatment for a PEx will also often include the modification of inhaled medications, aggressive chest physiotherapy and increased compliance with prescribed medications, all which could have contributed to the observed treatment effect. The study focused on preschool children; however, this narrow population characteristic may not be generalisable to older children with CF.

In conclusion, this study provides further evidence for the utility of LCI as a tool for clinical monitoring of young children with CF. While the commercial availability of MBW systems has made the implementation of this technique into clinical practice more feasible, capital costs for equipment, as well as personnel training and results interpretation are operational challenges that will have to be overcome. As we gain more insight into defining a minimal clinically important difference in LCI and how visitto-visit changes in LCI can be interpreted to guide clinical decisions, MBW testing may become an integral part of the clinical management of young patients with CF.

Acknowledgements The authors would like to thank the children and families who participated in this research study, as well as Hailey Webster, Miriam Davis, Robin C Johnson, Renee Jensen, Maria Ester Pizarro, Mica Kane, Charles C Clem and Leah Schornick for their role in recruiting participants, collecting and interpreting the MBW data.

Contributors All authors conceived of the study. SDD, GR-B and FR supervised data collection at their respective sites. JHR and SS analysed the data. JHR drafted the manuscript. All authors reviewed, revised and approved the final manuscript.

Funding This study was funded by the National Heart, Lung, and Blood Institute R01HL116232-04. JHR's fellowship was funded by Cystic Fibrosis Canada.

Competing interests None declared.

Ethics approval The study was approved by the Research Ethics Boards at the Hospital for Sick Children (REB \# 1000036303), Riley Children's Hospital (1401277863), and the Office of Human Research Ethics at University of North Carolina at Chapel Hill (13-1258).

Provenance and peer review Not commissioned; externally peer reviewed.

(C) Article author(s) (or their employer(s) unless otherwise stated in the text of the article) 2018. All rights reserved. No commercial use is permitted unless otherwise expressly granted.

\section{REFERENCES}

1 O'Sullivan BP, Freedman SD. Cystic fibrosis. Lancet 2009;373:1891-904.

2 Waters V, Stanojevic S, Atenafu EG, et al. Effect of pulmonary exacerbations on longterm lung function decline in cystic fibrosis. Eur Respir J 2012;40:61-6.

3 Sanders DB, Goss CH. Pulmonary exacerbations as indicators of progression of lung disease in young children with CF. Thorax 2013;68:608-9.

4 Liou TG, Adler FR, Fitzsimmons SC, et al. Predictive 5-year survivorship model of cystic fibrosis. Am J Epidemiol 2001;153:345-52.

5 Byrnes CA, Vidmar S, Cheney JL, et al. Prospective evaluation of respiratory exacerbations in children with cystic fibrosis from newborn screening to 5 years of age. Thorax 2013;68:643-51.

6 Stanojevic S, McDonald A, Waters V, et al. Effect of pulmonary exacerbations treated with oral antibiotics on clinical outcomes in cystic fibrosis. Thorax 2017;72:327-32

7 Wagener JS, Rasouliyan L, VanDevanter DR, et al. Oral, inhaled, and intravenous antibiotic choice for treating pulmonary exacerbations in cystic fibrosis. Pediatr Pulmonol 2013;48:666-73.

8 Fuchs HJ, Borowitz DS, Christiansen DH, et al. Effect of aerosolized recombinant human DNase on exacerbations of respiratory symptoms and on pulmonary function in patients with cystic fibrosis. The Pulmozyme Study Group. N Eng/ J Med 1994;331:637-42.

9 Rosenfeld M, Emerson J, Williams-Warren J, et al. Defining a pulmonary exacerbation in cystic fibrosis. J Pediatr 2001;139:359-65.

10 Waters V, Ratjen F. Pulmonary Exacerbations in Children with Cystic Fibrosis. Ann Am Thorac Soc 2015;12(Suppl 2):S200-6.

11 Anstead M, Saiman L, Mayer-Hamblett N, et al. Pulmonary exacerbations in CF patients with early lung disease. J Cyst Fibros 2014;13:74-9.

12 Monto AS, Ullman BM. Acute respiratory illness in an American community. The Tecumseh study. JAMA 1974;227:164-9. 
13 Brumback LC, Baines A, Ratjen F, et al. Pulmonary exacerbations and parent-reported outcomes in children $<6$ years with cystic fibrosis. Pediatr Pulmonol 2014.

14 Regelmann WE, Schechter MS, Wagener JS, et al. Pulmonary exacerbations in cystic fibrosis: young children with characteristic signs and symptoms. Pediatr Pulmonol 2013;48:649-57.

15 Schechter MS, Regelmann WE, Sawicki GS, et al. Antibiotic treatment of signs and symptoms of pulmonary exacerbations: a comparison by care site. Pediatr Pulmonol 2015:50:431-40.

16 Robinson PD, Latzin P, Verbanck $S$, et al. Consensus statement for inert gas washout measurement using multiple- and single- breath tests. Eur Respir $J$ 2013:41:507-22

17 Aurora P, Bush A, Gustafsson P, et al. Multiple-breath washout as a marker of lung disease in preschool children with cystic fibrosis. Am J Respir Crit Care Med 2005; 171:249-56.

18 Gustafsson PM, Aurora P, Lindblad A. Evaluation of ventilation maldistribution as an early indicator of lung disease in children with cystic fibrosis. Eur Respir J 2003;22:972-9.

19 Aurora P, Stanojevic S, Wade A, et al. Lung clearance index at 4 years predicts subsequent lung function in children with cystic fibrosis. Am J Respir Crit Care Med 2011;183:752-8.

20 Horsley AR, Davies JC, Gray RD, et al. Changes in physiological, functional and structural markers of cystic fibrosis lung disease with treatment of a pulmonary exacerbation. Thorax 2013;68:532-9.

21 Sonneveld N, Stanojevic S, Amin R, et al. Lung clearance index in cystic fibrosis subjects treated for pulmonary exacerbations. Eur Respir J 2015;46:1055-64.

22 Hatziagorou E, Avramidou V, Kirvassilis F, et al. Use of lung clearance index to assess the response to intravenous treatment in cystic fibrosis. Hippokratia 2015:19:47-52.

23 Welsh L, Nesci C, Tran H, et al. Lung clearance index during hospital admission in school-age children with cystic fibrosis. J Cyst Fibros 2014;13:687-91.
24 Robinson PD, Cooper P, Van Asperen P, et al. Using index of ventilation to assess response to treatment for acute pulmonary exacerbation in children with cystic fibrosis. Pediatr Pulmonol 2009;44:733-42.

25 Stahl M, Wielpütz MO, Graeber SY, et al. Comparison of Lung Clearance Index and Magnetic Resonance Imaging for Assessment of Lung Disease in Children with Cystic Fibrosis. Am J Respir Crit Care Med 2017;195:349-59.

26 Sanders DB, Bittner RC, Rosenfeld M, et al. Failure to recover to baseline pulmonary function after cystic fibrosis pulmonary exacerbation. Am J Respir Crit Care Med 2010;182:627-32

27 Waters V, Atenafu EG, Salazar JG, et al. Chronic Stenotrophomonas maltophilia infection and exacerbation outcomes in cystic fibrosis. J Cyst Fibros 2012;11:8-13.

28 Stanojevic S, Davis SD, Retsch-Bogart G, et al. Progression of Lung Disease in Preschool Patients with Cystic Fibrosis. Am J Respir Crit Care Med 2017; 195:1216-25.

29 Jensen R, Stanojevic S, Klingel M, et al. A Systematic Approach to Multiple Breath Nitrogen Washout Test Quality. PLoS One 2016;11:e0157523.

30 Beydon N, Davis SD, Lombardi E, et al. An official American Thoracic Society/European Respiratory Society statement: pulmonary function testing in preschool children. Am J Respir Crit Care Med 2007;175:1304-45.

31 Quanjer PH, Stanojevic S, Cole TJ, et al. Multi-ethnic reference values for spirometry for the 3-95-yr age range: the global lung function 2012 equations. Eur Respir J 2012;40:1324-43.

32 Oude Engberink E, Ratjen F, Davis SD, et al. Inter-test reproducibility of the lung clearance index measured by multiple breath washout. Eur Respir J 2017;50:1700433.

33 Grasemann $\mathrm{H}$, Ciet $\mathrm{P}$, Amin $\mathrm{R}$, et al. Changes in magnetic resonance imaging scores and ventilation inhomogeneity in children with cystic fibrosis pulmonary exacerbations. Eur Respir J 2017:50:1700244.

34 Yammine S, Bigler A, Casaulta C, et al. Reasons for heterogeneous change in $\mathrm{LCl}$ in children with cystic fibrosis after antibiotic treatment. Thorax 2014;69:183. 